\title{
Mitochondrial genotype and breast cancer predisposition
}

\author{
ANNA M. CZARNECKA ${ }^{1,2}$, TOMASZ KRAWCZYK ${ }^{3}$, KATARZYNA PLAK ${ }^{1}$, ALEKSANDRA KLEMBA ${ }^{1}$, \\ MAREK ZDROZNY ${ }^{4}$, REBECCA S. ARNOLD ${ }^{9}$, BARBARA KOFLER ${ }^{8}$, PAWEL GOLIK ${ }^{1,5}$, \\ ALEKSANDRA SZYBINSKA $^{6}$, JAN LUBINSKI ${ }^{7}$, MALGORZATA MOSSAKOWSKA ${ }^{6}$, \\ EWA BARTNIK ${ }^{1,5}$ and JOHN A. PETROS ${ }^{9-11}$
}

\begin{abstract}
${ }^{1}$ Institute of Genetics and Biotechnology, Faculty of Biology, University of Warsaw, Pawinskiego 5a, 02-106, Warsaw;
${ }^{2}$ Laboratory of Molecular Oncology, Department of Oncology, Szaserow 128, 04-141 Warsaw, ${ }^{3}$ Clinical Pathology Laboratory, Monument Institute of Polish Mothers Health Center, Lodz; ${ }^{4}$ Department of Oncological Surgery and Breast Diseases, Monument Institute of Polish Mothers Health Center, Lodz; ${ }^{5}$ Institute of Biochemistry and Biophysics, Polish Academy of Sciences, Pawinskiego 5a, 02-106 Warsaw; ${ }^{6}$ International Institute of Molecular and Cell Biology, Warszawa,

${ }^{7}$ International Hereditary Cancer Center, Department of Genetics and Pathology, Pomeranian Medical University, Szczecin, Poland; ${ }^{8}$ Department of Pediatrics, University Hospital Salzburg, Paracelsus Medical University, Müllner Hauptstrasse 48, A-5020 Salzburg, Austria; ${ }^{9}$ Department of Urology, Emory University School of Medicine, 1365 Clifton Road, Building B, Atlanta, GA 30322; ${ }^{10}$ The Atlanta VA Medical Center, 1670 Clairmont Road, Decatur, GA 30033;

${ }^{11}$ Department of Pathology and Laboratory Medicine, Emory University School of Medicine, Atlanta, GA 30322, USA
\end{abstract}

Received April 21,2010; Accepted May 31, 2010

DOI: 10.3892/or_00001014

\begin{abstract}
Breast cancer is the most commonly diagnosed cancer in women. Despite recent advances in breast cancer research, a comprehensive set of genetic markers of increased breast cancer risk remain elusive. Recently mitochondrial DNA (mtDNA) mutations have been found in many types of cancer, including breast cancer. To investigate the possible role of mitochondrial genetics in breast cancer predisposition and biology we analyzed the D-loop sequence of cancer patients and assigned mitochondrial haplogroup using RFLP analysis. We detected a significantly greater incidence of mtDNA polymorphisms T239C, A263G and C16207T and a significant lower incidence of A73G, C150T, T16183C, T16189C, C16223T, T16362C in patients with breast cancer compared to database controls. The mitochondrial haplogroup distribution in patients with breast cancer differs from a group of cancer-free controls and the general Polish population in that haplogroup I is over-represented in individuals with cancer. These findings suggest that mitochondrial haplogroup I as well as other polymorphic variants defined by SNPs in the D-loop may be associated with an increased risk of developing breast cancer.
\end{abstract}

Correspondence to: Dr John A. Petros, Department of Urology, Emory University School of Medicine, 1365 Clifton Road, Atlanta, GA 30322, USA

E-mail: jpetros@emory.edu

Key words: breast cancer, mitochondrial genotype

\section{Introduction}

An estimated 1.15 million new breast cancer cases are diagnosed worldwide per year (1). In the United States breast cancer is the most common cancer among women (over 200,000 cases annually) and the second most common cause of cancer mortality (over 40,000 deaths per year) accounting for $15 \%$ of cancer deaths among American women and 26\% of all new cancer cases among women $(2,3)$. Also in the European Union (EU) breast cancer is the the second most commonly diagnosed cancer and accounts for 17.9 deaths per 100,000 population (4). Multiple diagnostic and prognostic factors have been defined for breast cancer. Prognostic factors generally are related to surgical and pathological findings and include tumor grade, stage and lymph node metastasis status. Few predictive biomarkers are currently available. High throughput molecular technologies are reshaping our understanding of breast cancer and a molecular taxonomy that has stronger predictive power is slowly emerging. In some cases a molecular grading system has been proposed as in the case of ductal cancer in situ of the breast (5), and novel prognostic/predictive gene signatures have been identified (6).

There are likely many low-penetrance genes involved in breast cancer carcinogenesis, and their cumulative attributable risk for breast cancer development is substantial. Moreover, $5 \%$ of breast cancers are associated with a proven genetic predisposition, transmitted as an autosomal dominant trait. It is known that mutations in the BRCA1 or BRCA2 genes are associated with a high risk of breast or/and ovarian cancer. Women with BRCA1 and BRCA2 mutations have a $65-85 \%$ cumulative lifetime risk of developing invasive breast cancer and a $15-65 \%$ cumulative lifetime risk of developing 
invasive ovarian cancer (7-10). Other genes besides BRCA1 and BRCA2 related to breast cancer susceptibility include the 'guardian of the genome' TP53, and genes that encode proteins in p53-DNA repair-pathways, the PTEN (phosphatase and tensin homolog-mutated in multiple advanced cancers 1) gene, and the CHEK2 (protein kinase CHK2 isoform c) gene (10-12).

Other genes mutated in breast cancer patients include the ATM (ataxia telangiectasia mutated) gene, XPD (ERCC2, excision repair cross-complementing rodent repair deficiency, complementation group 2 protein) and HER-2 (human epidermal growth factor receptor 2$)$ gene $(13,14)$. Susceptibility alleles have been located in the genes PALB2 (15), STK11/ $L K B 1$, and MSH2/MLH1 (1). Recently, genome-wide single nucleotide polymorphism (SNP) association studies revealed genetic risk variants independent of family history in loci of: FGFR2, TNRC9, MAP3K1, LSP1, 2q35, 5p12, 8q24, followed by CASP8, FGFR2, TNRC9, MAP3K1, LSP1, and rs 13387042 on $2 q 35, r s 313281615$ on $8 q 24$, and $r s 10941679$ on 5 p 12 for individuals of European ancestry $(1,16,17)$. It is likely that additional genetic loci contribute to breast cancer susceptibility. In this regard, mitochondrial genetics may be important in carcinogenesis $(18,19)$ and is an important area in oncology research (20-22). Breast nipple aspirate fluid (NAF) with mtDNA mutations at positions 204, 207 and 16293 has been associated with breast cancer (23) and mtDNA D-loop mutations have been proposed as an independent prognostic marker in breast cancer (24).

At least $50 \%$ of women will receive a false-positive result after having annual screening mammograms for a decade, and almost $20 \%$ of women will undergo a biopsy for that reason. Also, nearly $25 \%$ of women will have a false-positive test at some point during 10 years of regular clinical breast examinations (25). Data from The National Cancer Institute (NCI) puts the false negative rate of mammography as high as $40 \%$ for women aged $40-49$ and $10 \%$ in women over 50 . Breast tissue is more dense among younger women, making it more difficult to detect tumors. For this reason, false negatives are twice as likely to occur in premenopausal mammograms (26). Ultrasound is not used for routine breast cancer screening because it does not consistently detect certain early signs of cancer such as microcalcifications, but mammography alone has a cancer detection rate of 7.6 women per 1,000 women screened and adding an ultrasound increases the rate to 11.8 women per 1,000 women screened, an increase of $28 \%$ (27). Several risk assessment tools, such as BRCAPRO, the Claus model, and the Tyrer-Cuzick model, are available to help health professionals estimate a woman's breast cancer risk. These tools give approximate, rather than precise, estimates of breast cancer risk based on different combinations of risk factors (28). Because studies of the etiology of breast cancer have failed to identify feasible primary prevention strategies suitable for use in the general population, reducing mortality from breast cancer through early detection has become a high priority. The potential for reducing death rates from breast cancer is contingent upon increasing mammography screening rates and subsequently detecting the disease at an early stage, when more treatment options are available and survival rates are higher. Therefore, additional accurate genetic markers of increased risk would be valuable (29).
There is an association between somatic mtDNA mutations and cancer development, progression and metastasis (18,21,30-37). In addition, recent studies have shown that inherited polymorphisms or mutations of the mitochondrial genome may modulate the risk of developing cancer, including prostate, oral and colorectal cancers (18,38-41). Somatic mtDNA mutations have been described in various types of human cancers including bladder, brain, breast, colon, head and neck, lung, ovaries, prostate, thyroid, and breast cancers $(20,21,31,33)$. At the same time inherited polymorphisms have been identified as contributing factors in cancer development $(35,39,40)$.

Our current research builds upon other studies indicating that mtDNA mutations may be more powerful in detecting tumor cells in bodily fluids and cytological specimens than mutations found in nuclear DNA $(34,41)$. Fliss and colleagues (42) have reported facile detection of mtDNA mutations in diagnostic samples, reliably detecting known mutations of mtDNA against the background of normal mtDNAs present in diagnostic specimens.

In the present study, we examined the genetic alterations in the D-loop region of mtDNA in primary human breast cancer tissues and paired control tissues. We have also investigated the distribution of haplogroups in the patient cohort, in an effort to address the significance of mtDNA alterations and inherited polymorphisms in breast tumorigenesis. In order to test for association with cancer susceptibility and frequency of mtDNA variants in BC patients we analysed the frequency of mtDNA polymorphisms and compared it with the frequency of those polymorphisms in healthy controls, centenarians and the general Polish $(43)$ and European $(44,45)$ populations.

\section{Materials and methods}

Patients. We studied 44 breast cancer (BC) patients who had undergone surgery (total mastectomy) at the Monument Institute of Polish Mothers Health Center, Lodz, Poland. Adjacent benign tissue was available in all cases. The patients received no chemotherapy, radiotherapy, or hormonal therapy before surgery. Histological analysis was based on the standard AJCC classification (46). For each specimen we determined histological type and grade, depth of infiltration, estrogen receptor (ER), progesterone receptor (PR) expression and the presence or absence of metastases in the lymph nodes.

The cancers were predominantly stage T1 including 5 pT1b patients, and 19 pT1c patients. Eighteen women were diagnosed with stage pT2 disease. Twenty-three patients were lymph node negative while 20 were node positive (all pN1) and one patient was Nx. Estrogen receptor (ER) and progesterone receptor (PR) staining were as follows: $\mathrm{ER}(0), 16 ; \mathrm{ER}(+), 17 ; \mathrm{ER}(++), 2 ; \mathrm{ER}(+++), 9 ; \mathrm{PR}(0), 15$, $\mathrm{PR}(+), 16 ; \mathrm{PR}(++), 3 ; \mathrm{PR}(+++), 10$. The mean age of the breast cancer population was 57 , median age was 58 .

The healthy cohort (control group 1) of 100 individuals was recruited at the Department of Genetics and Pathology, International Hereditary Cancer Center, Pomeranian Medical University. The healthy cohort consisted of healthy females with a negative family history of cancer. The females were part of a population-based study of 1.5 million Polish residents designed to identify familial aggregations of cancer by the 


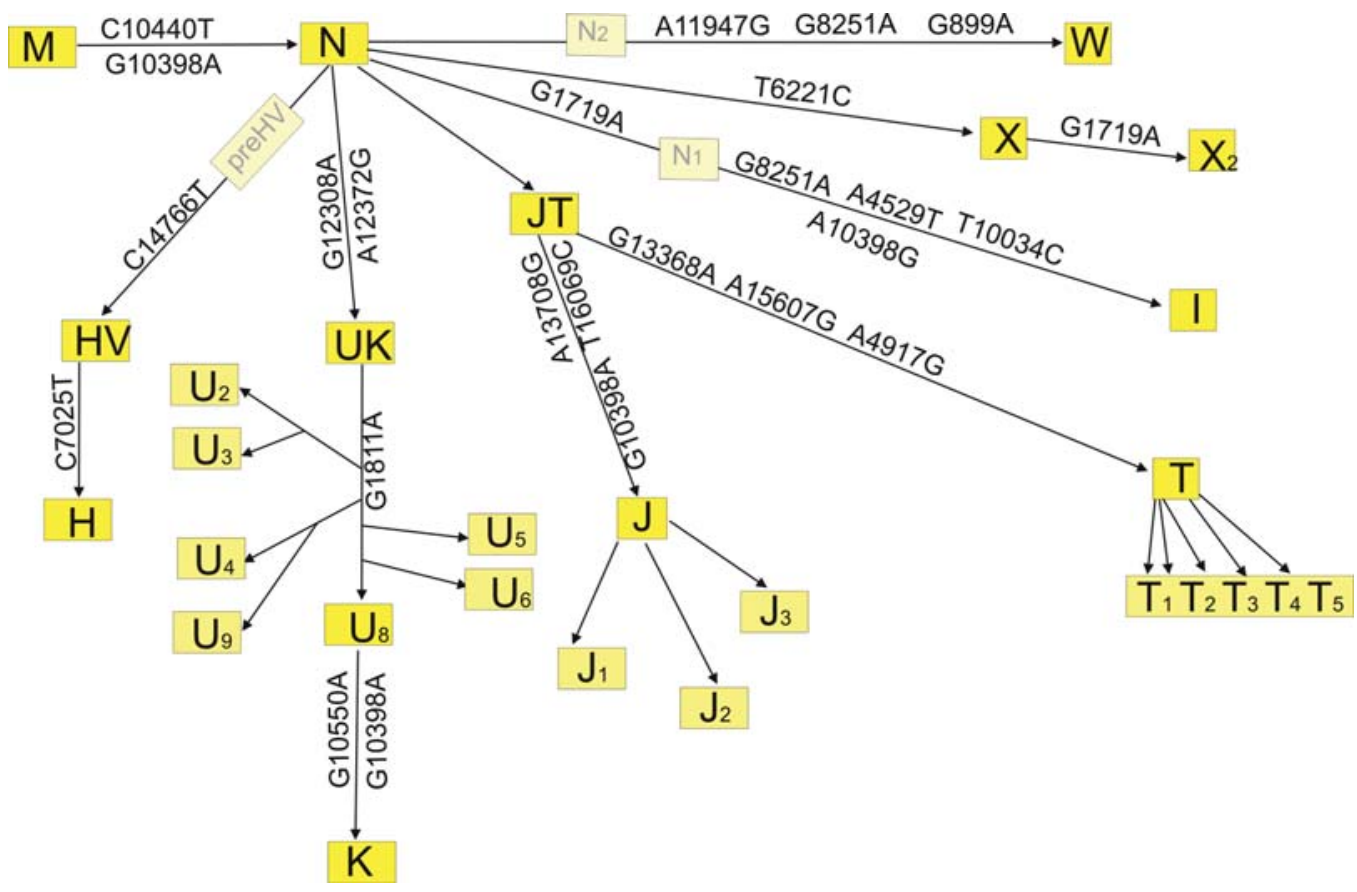

Figure 1. Haplogroup assignement pattern.

International Hereditary Cancer Center. These controls were interviewed in 2007. Women affected with any malignancy, or with any cancers diagnosed in a first-degree relative were excluded from this control group. The mean and median age of this healthy, breast cancer-free population was 55 years. Thus, the healthy control group is both free of breast cancer and unlikely to have inherited a genetic predisposition to the development of breast cancer.

One hundred and twenty-six centenarians (control group 2) with negative cancer history and negative family history of cancer were recruited from Polish Centenarians project of the International Institute of Molecular and Cell Biology in Warsaw (47). The control and general Polish populations have been described previously (43). All three populations share the same ethnicity, nationality, parentage, ancestry and currently reside in Poland. This study did not include any patients of Asian, African or Jewish origin.

The project was approved by the local Ethics Committee at the Medical University of Warsaw, Warsaw, Poland (KB0/6/2007 to AMC). The Centenarians database was registered at the Bureau of the Inspector General for the Protection of Personal Data in May 1999. An agreement from the Bioethical Committee at the Central Clinical Hospital of the Military Medical Academy in Warsaw was granted for the Polish Centenarians Program. The centenarians or their relatives signed an informed consent for participation in the project (47). The Hereditary Cancer Registry at the Pomeranian Medical University in Szczecin, Poland contains clinical and epidemiological data collected since 1997, and was approved by the Ethics Committee of Pomeranian Medical University in Szczecin, Poland and all participants gave informed consent prior to enrolling in the study. All women received genetic counseling prior to and at the provision of their test results (48).
Tissue collection and DNA isolation. Tissue obtained at surgery was sub-divided into two parts. One portion was fixed in buffered formalin ( $\mathrm{pH}$ 7.4) for routine histopathological assessment, while the rest was immediately frozen in liquid nitrogen and stored at $-80^{\circ} \mathrm{C}$. Non-neoplastic material was also collected at surgery and stored at $-80^{\circ} \mathrm{C}$ until assayed.

DNA isolation. Cancer and matched normal tissue DNA was isolated using a PureGene DNA Purification Kit (Gentra Systems) according to the manufacturer's specifications. Control group DNA was isolated using phenol/ chloroform extraction and ethanol precipitation from $10 \mathrm{ml}$ blood samples. Centenarians' DNA was isolated from lymphoblast cell culture with NucleoSpin Blood L (MacheryNagel) according to the manufacturer's specifications. We have previously demonstrated that haplotype-defining SNPs may be determined on either blood or tissue DNA without changing haplotype assignment (49).

Polymorphism analysis. The mtDNA in normal tissue of individual patients was used to assign that individual's mitochondrial haplotype when different from the reference sequence and that difference was defined as a germline (inherited) polymorphism. The revised Cambridge sequence (CRS) (50) of the relevant position, in each case, is shown for reference in Table I.

Haplogroup analysis by RLFP. Common polymorphisms in mtDNA determine classes of related genotypes, referred to as haplogroups. These were detected by restriction fragment length polymorphism (RFLP) analysis. Haplogroup RLFP analysis, restriction enzymes and primers used are summarized in Fig. 1 and Table I. 
Table I. RFLP pattern used to establish haplogroups.

\begin{tabular}{|c|c|c|c|c|c|c|c|c|c|c|}
\hline \multirow[b]{2}{*}{ Restriction site } & \multicolumn{10}{|c|}{ Haplogroup } \\
\hline & $\mathrm{H}$ & I & $\mathrm{J}$ & $\mathrm{K}$ & $\mathrm{T}$ & $\mathrm{U}$ & $\mathrm{V}$ & $\mathrm{W}$ & $\mathrm{X}$ & M \\
\hline -1715 Dde I & & + & & & & & & & + & \\
\hline -1806 Psi I & + & & & & & + & + & & & \\
\hline +4220 Nla III & + & & + & & + & & & & & \\
\hline-4529 Hae II & & + & & & & & & & & \\
\hline-4577 Nla III & & & & & & & + & & & \\
\hline-4915 Bfa I & & + & & & + & & & & & \\
\hline +6230 Mnl I & & & & & + & & & & + & \\
\hline -7015 Alu I & + & & & & & & & & & \\
\hline +8249 Ava II & & + & & & & & & + & & \\
\hline -8994 Hae III & & & & & & & & + & & \\
\hline -9052 Hae II & & & & + & & & & & & \\
\hline +10028 Alu I & & + & & & & & & & & \\
\hline +10394 Dde I & & + & + & + & & & & & & + \\
\hline +10397 Alu I & & & & & & & & & & + \\
\hline +10497 Nla III & & & & + & & & & & & \\
\hline +11949 BsF I & & + & & & & & & + & & \\
\hline +12308 Hinf I & & & & + & & + & & & & \\
\hline +13366 Bam HI & & & & & + & & & & & \\
\hline -13704 Bst NI & & & + & & & & & & & \\
\hline-14766 Mse I & + & & & & & & + & & & \\
\hline +15606 Alu I & & & & & + & & & & & \\
\hline -15606 Alu I & & & & & & & & & & \\
\hline +15904 Mse I & & & & & & & + & & & \\
\hline-15925 Msp I & & & & & + & & & & & \\
\hline-16065 Hinf I & & & + & & & & & & & \\
\hline +16389 Bam HI & & + & & & & & & & & \\
\hline
\end{tabular}

Haplogroup analysis by multiplex-PCR/sequencing. To verify haplogroups established by RFLP (as in Table I) a multiplex$\mathrm{PCR} /$ primer extension analysis as described previously was performed (51). Moreover, haplogroups were also assigned based on specific D-loop polymorphisms according to concensus data (52-54). If any non-specific RFLP or multiplex$\mathrm{PCR} /$ primer extension variants were found, sequencing with appropriate primers listed in Table I was performed in order to unambiguously determine the polymorphism.

PCR amplification of a D-loop segment of mtDNA. MtDNA fragments (15587-964) spanning the D-loop region (spanning nucleotide 16024 to 576) were amplified using eight pairs of primers. The primer pairs used and the sizes of the amplified products are shown in Table II.

Fifty-microlitre reactions contained $10 \mathrm{ng}$ DNA and $0.5 \mu \mathrm{M}$ primers, $0.2 \mathrm{mM}$ of each deoxynucleotide triphosphate (dNTP), $1 \mathrm{U}$ of FIREPol ${ }^{\circledR}$ DNA Polymerase (Solis BioDyne, Estonia) or Pfu DNA Polymerase (Fermentas AB, Lithuania) and $2.5 \mathrm{mM} \mathrm{MgCl}$. DNA was subjected to the following cycling conditions: initial denaturing at $95^{\circ} \mathrm{C}$ for $3 \mathrm{~min}$ followed by $94^{\circ} \mathrm{C}$ for $1 \mathrm{~min}, 55^{\circ} \mathrm{C}$ for $30 \mathrm{sec}$, and $72^{\circ} \mathrm{C}$ for $1 \mathrm{~min}$ for 40 cycles and final extension step at $72^{\circ} \mathrm{C}$ for $7 \mathrm{~min}$. Two microlitres of PCR products were analysed on an ethidium bromide-stained, $3 \%$ agarose gel (40 min at $70 \mathrm{~V}$ ) to demonstrate the presence of the amplification product and for its quantification.

$m t D N A$ sequence analysis. Sequence analysis was performed by: FinchTV Version 1.4.0 (Geospiza Inc., USA) and BioEdit version 7.0.5.3 (Copyright Tom Hall 1999-2007), contig assembly was performed with Sequencher 4.1.4 (Gene Codes Corp., Ann Arbor, MI USA) and multiple sequence alignment was performed with Clustal W (55). Normal and cancer tissue mtDNA sequences were compared with the revised Cambridge Reference Sequence (CRS) and sequence variants were recorded $(50,56)$.

Statistical analysis. Two tailed non-directional Fisher-Irwin (Fisher's exact test) was used for statistical analysis (57). Statistical analysis was performed with PAST-PAlaeontological STatistics, ver. 1.34 and Analyse-it for Microsoft Excel General and Clinical Laboratory modules Version 1.73 [Analyse-it Software, Ltd., Copyright ${ }^{\circledR}$ 1997-2005]. 
Table II. Sequences of primers used for mtDNA D-loop sequencing (listed according to start position in mtDNA).

\begin{tabular}{llc}
\hline Primer & \multicolumn{1}{c}{ Primer sequence $\left(5^{\prime}-3^{\prime}\right)$} & Amplified mtDNA region $($ CRS $)$ \\
\hline $108 \mathrm{~F}$ & AGCACCCTATGTCGCAGTATC & $108-638$ \\
$276 \mathrm{R}$ & TCTGTGTGGAAAGTGGCTGTG & $16344-276$ \\
$315 \mathrm{~F}$ & CGCTTCTGGCCACAGCAC & $315-803$ \\
$548 \mathrm{~F}$ & CCAACCAAACCCCAAAGAC & $548-964$ \\
$559 \mathrm{R}$ & GGGTTTGGTTGGTCCGGG & $16495-559$ \\
$638 \mathrm{R}$ & GGTGATGTGAGCCCGTCTAAAC & $108-638$ \\
$803 \mathrm{R}$ & GGTGTGGCTAGGCTAAGC & $315-803$ \\
$964 \mathrm{R}$ & GGGAGGGGGTGATCTAAAAC & $548-964$ \\
$15587 \mathrm{~F}$ & CTCCGATCCGTCCCTAACAAAC & $15587-16185$ \\
$15879 \mathrm{~F}$ & AATGGGCCTGTCCTTGTAG & $15879-16545$ \\
$16098 \mathrm{~F}$ & ACATTACTGCCAGCCACCATG & $16098-16456$ \\
$16185 \mathrm{R}$ & GGTTTTGATGTGGATTGGGT & $15587-16185$ \\
$16344 \mathrm{~F}$ & CAGTCAAATCCCTTCTCGTCCC & $16344-276$ \\
$16456 \mathrm{R}$ & CCGGAGCGAGGAGAGTAGC & $16098-16456$ \\
$16495 \mathrm{~F}$ & CGACATCTGGTTCCTACTTC & $16495-559$ \\
$16545 \mathrm{R}$ & AACGTGTGGGCTATTTAGGC & $15879-16545$ \\
\hline
\end{tabular}

The difference was considered statistically significant at $\mathrm{p}<0.05$. In selected cases, to confirm the result of Fisher's test, Yates's $\chi$ and uncorrected $\chi^{2}$ test ('N-1' $\chi^{2}$ test) were used as expected to give relatively low type I error in case of a small research cohort. The statistics were performed according to published statistical standards (57). To further understand the significance of specific polymorphisms as factors for favorable outcomes (odds ratio, relative risk, difference in proportions, absolute and relative reduction in risk) and of the effectiveness of a diagnostic criterium (number needed to diagnose, specificity, positive and negative predictive values, positive and negative likelihood ratios, diagnostic and error odds ratios) additional analysis was performed. The parameters, as well as the confidence intervals for the estimated parameters are computed by using standard definitions and methods $(59,60)$.

\section{Results}

In order to test the hypothesis that the inherited mitochondrial genotype of women with breast cancer is different from controls, several comparisons were made. The standard 9haplogroup RFLP analysis of the patients with breast cancer was compared to a carefully selected breast cancer-free control group of 100 (control group 1), 101 centenarians (control group 2) and the general Polish population (described previously). To further determine whether the inherited SNP profile was different than population controls, we performed D-loop sequencing and compared the results to the frequencies found in the on line searchable database $\mathrm{mtDB}$.

Haplogroup distributions differs breast cancer patients. Altogether, our analysis of 44 breast cancer (BC) patients has shown that $16(36 \%)$ belong to haplogroup H, 6 (14\%) to haplogroup I, 5 (11\%) to haplogroups $\mathrm{K}$ and V, $3(7 \%)$ to haplogroup T, $2(5 \%)$ to haplogroups J, U and W, $1(2 \%)$ to $\mathrm{X}$. In $2(4 \%)$ cases, no specific haplogroup was assessed due to non-specific polymorphisms in haplogroup-defining positions (Fig. 2). Control group 1 (healthy cancer-free woman) were $50 \% \mathrm{H}, 6 \% \mathrm{I}, 13 \% \mathrm{~J}, 6 \% \mathrm{~K}, 8 \% \mathrm{~T}, 0 \% \mathrm{U}, 15 \%$ $\mathrm{V}, 1 \% \mathrm{~W}$ and $1 \% \mathrm{X}$. Statistical analysis revealed also that haplogroup distributions of the $\mathrm{BC}$ cohort and general Polish population is different (Table III; Fig. 1). Most striking is the over-representation of haplogroup I individuals among BC patients (14 vs. $3 \%$; $\mathrm{p}=0.017$ ) in comparison to the general Polish population. The significance of the cancer specific haplogroup distribution is even more pronounced if the cancer cohort is compared to a large Polish cohort $(n=436)$ haplogroup distribution, 14 vs. $2 \%$; $(p=0.001)$ for haplogroup I (61). Nevertheless, this haplogroup is characterized by many RFLP-markers and no single mtDNA position may serve as a cancer marker. Therefore, it is possible that the interactions of multiple mitochondrial DNA variant modify breast cancer risk in the case of haplogroup I (62), possibly with special role of A10398G polymorphism $(35,37)$. Other haplogroups seem to be represented in the cancer population at a frequency similar to the general Polish population.

At the same time BC cohort individuals if compared to centenarians carry haplogroup $\mathrm{H}$ less frequently (38 vs. $58 \%$; $=0.019)$. Since polymorphism T7028C defines haplogroup $\mathrm{H}$ (7028C), therefore, it seems that $7028 \mathrm{C}$ variant may be classified as protective against cancer development. It is for the reason that centenarians carry the $7028 \mathrm{C} 58 \%$ of the time while in the breast cancer group patients inherited the $7028 \mathrm{C} \mathrm{SNP} 36 \%$ of the time (43). This difference is statistically significant $\mathrm{p}=0.019$ with Fisher's exact test, which was confirmed both by Yates corrected $\chi^{2}, 5.118$ and $\mathrm{p}=0.024$ and 'N-1' $\chi^{2},{ }^{\prime}-1$ ' $\chi^{2}, 5.93$, and $\mathrm{p}=0.01$. These tests have been used as expected to give relatively low type I error. 


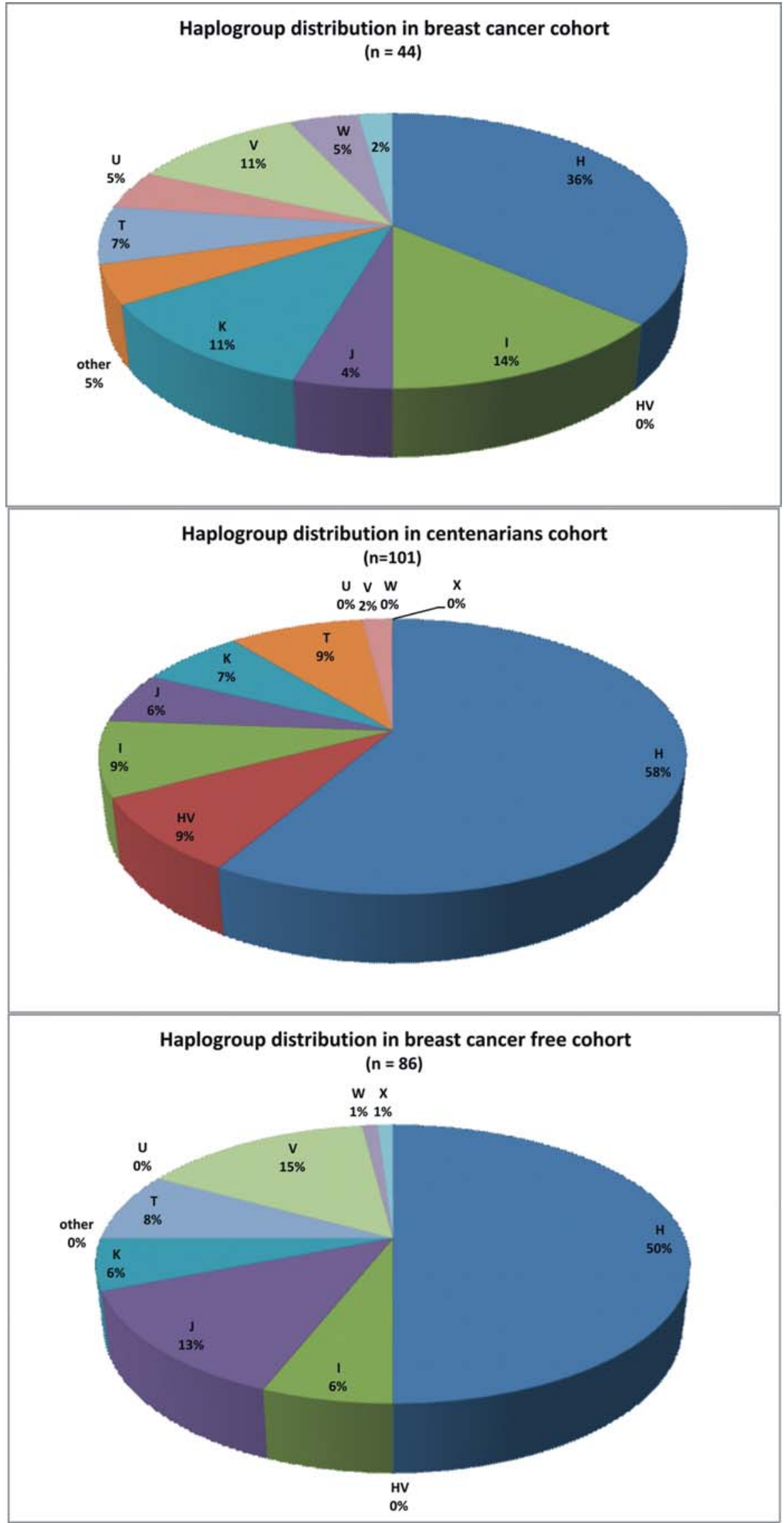

Figure 2. Haplogroup distribution in breast cancer, centenarians and healthy woman cohorts.

The statistics was performed as previously suggested (57). Moreover, in centenarians $7028 \mathrm{C}$ is found at relative risk
$\mathrm{RR}=1.31(95 \%$ CI: $1.0473<\mathrm{R} . \mathrm{R} .<1.6414)$, which is $31 \%$ more often than in the cancer cohort. This gives odds ratio 
Table III. Germ-line polymorphisms in the D-loop region of mtDNA of the breast cancer patients.

\begin{tabular}{|c|c|c|c|c|c|c|}
\hline $\begin{array}{l}\text { mtDNA } \\
\text { position } \\
(\mathrm{CRS})\end{array}$ & Polymorphism & No & $\begin{array}{l}\mathrm{A} / \mathrm{G} / \mathrm{C} / \mathrm{T} / \mathrm{del} \\
\text { frequency } \\
(\mathrm{mtDB})\end{array}$ & $\begin{array}{l}\text { Tissues where } \\
\text { sequence was found } \\
\text { (MITOMAP) }\end{array}$ & P-value & $\begin{array}{l}\text { Region/population where } \\
\text { sequence variant } \\
\text { predominantly was found }\end{array}$ \\
\hline 64 & $\mathrm{~T} \rightarrow \mathrm{C}$ & 1 & $0 / 0 / \mathbf{1 8 3 8} / 27$ & Aging brains & 0.482 & Africa, Japan, Autralia \\
\hline 73 & $\mathrm{~A} \rightarrow \mathrm{G}$ & 16 & $309 / \mathbf{1 5 5 5} / 1 / 0$ & $\begin{array}{l}\text { Aging brains, POLG/PEO } \\
\text { and control muscle, thyroid } \\
\text { tumor, oral cancer }\end{array}$ & $0.001 \mathrm{U}$ & Very common \\
\hline 146 & $\mathrm{~T} \rightarrow \mathrm{C}$ & 2 & $1 / 0 / \mathbf{1 9 0} / 1674$ & $\begin{array}{l}\text { Elderly fibroblasts, aging/AD } \\
\text { brains, POLG/PEO and control } \\
\text { muscle, prostate tumor, ovarian } \\
\text { cancer }\end{array}$ & 0.310 & $\begin{array}{l}\text { Africa, Japan, Taiwan, Finland } \\
\text { Italy, Spain, Algerian Jew, } \\
\text { India, Polynesia, Caucasian }\end{array}$ \\
\hline 150 & $\mathrm{C} \rightarrow \mathrm{T}$ & 2 & $0 / 2 / \mathbf{1 6 1 6} / 247$ & $\begin{array}{l}\text { Elderly fibroblasts/leukocytes, } \\
\text { lung tumor, thyroid tumor }\end{array}$ & $0.001 \mathrm{U}$ & China, Japan, Berbers, Italy \\
\hline 152 & $\mathrm{~T} \rightarrow \mathrm{C}$ & 4 & 0/0/396/1469 & $\begin{array}{l}\text { Aging brains, elderly fibroblasts, } \\
\text { ovarian cancer, oral cancer }\end{array}$ & 0.059 & $\begin{array}{l}\text { Africa, China, Japan, American, } \\
\text { Finland, Italy }\end{array}$ \\
\hline 153 & $\mathrm{~A} \rightarrow \mathrm{G}$ & 1 & $\begin{array}{c}\text { 1840/25/0/0 } \\
\text { Top of Form } \\
\text { Bottom of From }\end{array}$ & Polymorphism & 0.457 & Japan, Finland, Italy \\
\hline 189 & $\mathrm{~A} \rightarrow \mathrm{G}$ & 2 & $1782 / 75 / 8 / 0$ & $\begin{array}{l}\text { Elderly muscle, POLG/PEO } \\
\text { muscle and fibroblasts, aging } \\
\text { brains, prostate tumor }\end{array}$ & 0.697 & Japan, Finland, India \\
\hline 195 & $\mathrm{~T} \rightarrow \mathrm{C}$ & 4 & $11 / 0 / \mathbf{2 8 0} / 1574$ & $\begin{array}{l}\text { Elderly fibroblasts, lung-cancer } \\
\text { cells, aging/AD brains, thyroid } \\
\text { tumor, oral cancer }\end{array}$ & 0.301 & $\begin{array}{l}\text { Africa, Japan, American, } \\
\text { Finland, Italy, Caucasian }\end{array}$ \\
\hline 199 & $\mathrm{~T} \rightarrow \mathrm{C}$ & 1 & $0 / 0 / \mathbf{1 2 1} / 1744$ & $\begin{array}{l}\text { Ovarian cancer, } \\
\text { POLG/MNGIE muscle }\end{array}$ & 0.361 & China, Japan, Finland, India \\
\hline 204 & $\mathrm{~T} \rightarrow \mathrm{C}$ & 3 & $0 / 0 / \mathbf{1 2 3} / 1741$ & Oral cancer, prostate tumor & 0.765 & Japan, Finland, India \\
\hline 207 & $\mathrm{G} \rightarrow \mathrm{A}$ & 3 & $123 / 1741 / 0 / 0$ & $\begin{array}{l}\text { Oral cancer, prostate tumor, } \\
\text { thyroid tumor }\end{array}$ & 0.765 & Japan, Finland, India \\
\hline 225 & $\mathrm{G} \rightarrow \mathrm{A}$ & 1 & 10/1855/0/0 & Polymorphism & 0.227 & \\
\hline 226 & $\mathrm{~T} \rightarrow \mathrm{C}$ & 1 & $0 / 0 / 21 / 1863$ & Oral cancer & 0.400 & \\
\hline 228 & $\mathrm{G} \rightarrow \mathrm{A}$ & 1 & $\mathbf{5 7 / 1 8 0 5 / 0 / 3}$ & Polymorphism & 1.00 & $\begin{array}{l}\text { American, Finland, India, } \\
\text { Caucasian }\end{array}$ \\
\hline 239 & $\mathrm{~T} \rightarrow \mathrm{C}$ & 3 & $0 / 0 / 4 / 1861$ & $\begin{array}{l}\text { POLG/PEO muscle, ovarian } \\
\text { tumor, oral cancer }\end{array}$ & 0.0010 & Extremely rare, Italy \\
\hline 242 & $\mathrm{C} \rightarrow \mathrm{T}$ & 1 & $0 / 0 / 1854 / \mathbf{1 1}$ & POLG/PEO muscle & 0.245 & $\begin{array}{l}\text { Extremely rare, American, } \\
\text { Finland }\end{array}$ \\
\hline 250 & $\mathrm{~T} \rightarrow \mathrm{C}$ & 1 & $0 / 0 / \mathbf{1 7} / 1848$ & Oral cancer & 0.344 & $\begin{array}{l}\text { Africa, Japan, Taiwan, Finland, } \\
\text { Italy, Spain, Algerian Jew, } \\
\text { India, Polynesia, Caucasian }\end{array}$ \\
\hline 263 & $\mathrm{~A} \rightarrow \mathrm{G}$ & 32 & $6 / \mathbf{1 8 6 1} / 0 / 0$ & $\begin{array}{l}\text { POLG/MNGIE muscle, } \\
\text { oral cancer }\end{array}$ & 0.0010 & $\begin{array}{l}\text { Africa, Japan, China, Australia, } \\
\text { American, Finland, India }\end{array}$ \\
\hline 295 & $\mathrm{C} \rightarrow \mathrm{T}$ & 2 & $4 / 0 / 1788 / 75$ & POLG/MNGIE muscle & 0.697 & $\begin{array}{l}\text { American, Finland, India, } \\
\text { Caucasian }\end{array}$ \\
\hline 303 & $\begin{array}{l}\mathrm{C} 7 \rightarrow \mathrm{C} 8 \\
\text { (ins) }\end{array}$ & 15 & $\mathrm{Nn}$ & Multiple tumor types & - & $\begin{array}{l}\text { Africa, Japan, Taiwan, Finland, } \\
\text { Italy, Spain, India, Polynesia, } \\
\text { Caucasian, Ashkenazi Jew, } \\
\text { American, Australia }\end{array}$ \\
\hline $\begin{array}{l}310 \\
\text { (ins) }\end{array}$ & $\mathrm{C} 5 \rightarrow \mathrm{C} 6$ & 32 & $\mathrm{Nn}$ & Multiple tumor types & - & $\begin{array}{l}\text { Africa, Japan, Taiwan, Finland, } \\
\text { Italy, Spain, India, Polynesia, } \\
\text { Caucasian, Ashkenazi Jew, } \\
\text { American, Australia }\end{array}$ \\
\hline 462 & $\mathrm{C} \rightarrow \mathrm{T}$ & 1 & $0 / 0 / 2073 / 71$ & Thyroid tumor & 1.00 & $\begin{array}{l}\text { American, Finland, India, } \\
\text { Caucasian }\end{array}$ \\
\hline
\end{tabular}


Table III. Continued.

\begin{tabular}{|c|c|c|c|c|c|c|}
\hline $\begin{array}{l}\text { mtDNA } \\
\text { position } \\
(\mathrm{CRS})\end{array}$ & Polymorphism & No & $\begin{array}{l}\mathrm{A} / \mathrm{G} / \mathrm{C} / \mathrm{T} / \mathrm{del} \\
\text { frequency } \\
(\mathrm{mtDB})\end{array}$ & $\begin{array}{l}\text { Tissues where } \\
\text { sequence was found } \\
\text { (MITOMAP) }\end{array}$ & P-value & $\begin{array}{l}\text { Region/population where } \\
\text { sequence variant } \\
\text { predominantly was found }\end{array}$ \\
\hline 477 & $\mathrm{~T} \rightarrow \mathrm{C}$ & 1 & $0 / 1 / \mathbf{1 9} / 2124$ & AD brains, ovarian tumor & 0.335 & $\begin{array}{l}\text { Africa, China, Japan, American, } \\
\text { Finland, Italy, India }\end{array}$ \\
\hline 513 & $\begin{array}{l}\mathrm{A} \rightarrow \mathrm{AA} \\
\text { (ins) }\end{array}$ & 4 & $\mathrm{Nn}$ & - & - & $\mathrm{Nn}$ \\
\hline 514 & delC & 5 & $\mathrm{Nn}$ & $\begin{array}{l}\text { Head/neck tumor, ovarian cancer } \\
\text { and control tissue, thyroid, } \\
\text { prostate and breast tumors }\end{array}$ & & $\mathrm{Nn}$ \\
\hline 515 & $\operatorname{del} A$ & 3 & $\mathrm{Nn}$ & - & - & $\mathrm{Nn}$ \\
\hline 16111 & $\mathrm{C} \rightarrow \mathrm{T}$ & 1 & $1 / 0 / 1830 / 36$ & $\begin{array}{l}\text { Colonic crypts somatic mutations, } \\
\text { oral cancer }\end{array}$ & 0.581 & Japan \\
\hline 16126 & $\mathrm{~T} \rightarrow \mathrm{C}$ & 3 & 0/0/166/1701 & Oral cancer & 1.00 & $\begin{array}{l}\text { China, Japan, American, } \\
\text { Finland, Italy, India }\end{array}$ \\
\hline 16136 & $\mathrm{~T} \rightarrow \mathrm{C}$ & 1 & $0 / 0 / 24 / 1843$ & Polymorphism & 0.443 & Very rare Japan, Melanesia \\
\hline 16172 & $\mathrm{~T} \rightarrow \mathrm{C}$ & 1 & $0 / 0 / \mathbf{1 5 0} / 1717$ & $\begin{array}{l}\text { MNGIE tissues, head/neck tumor } \\
\text { back-mutation, oral cancer }\end{array}$ & 0.253 & Japan, Morocco, Finland, Italy \\
\hline 16183 & $\mathrm{~T} \rightarrow \mathrm{C}$ & 1 & $\begin{array}{c}1541 / 12 / 237 / \\
0 / 77\end{array}$ & $\begin{array}{l}\text { Lung tumor back-mutation, } \\
\text { prostate tumor }\end{array}$ & $0.036 \mathrm{U}$ & $\begin{array}{l}\text { China, Japan, Finland, Italy, } \\
\text { India }\end{array}$ \\
\hline 16189 & $\mathrm{~T} \rightarrow \mathrm{C}$ & 4 & $0 / 0 / \mathbf{5 2 2} / 1345$ & Prostate tumor & $0.004 \mathrm{U}$ & $\begin{array}{l}\text { Africa, Japan, India, Italy, } \\
\text { Finland }\end{array}$ \\
\hline 16192 & $\mathrm{C} \rightarrow \mathrm{T}$ & 3 & 0/0/1808/59 & Oral cancer & 0.169 & Rare, Japan, Finland, Italy \\
\hline 16207 & $\mathrm{C} \rightarrow \mathrm{T}$ & 1 & $1863 / 4 / 0 / 0$ & Polymorphism & 0.0230 & $\mathrm{Nn}$ \\
\hline 16222 & $\mathrm{C} \rightarrow \mathrm{T}$ & 1 & $0 / 0 / 1852 / \mathbf{1 5}$ & Polymorphism & 0.312 & Maroco, India, America \\
\hline 16223 & $\mathrm{C} \rightarrow \mathrm{T}$ & 2 & 0/0/992/875 & Oral cancer & $0.001 \mathrm{U}$ & $\begin{array}{l}\text { Africa, Japan, China, Australia, } \\
\text { India, Finland, Ashkenazi Jews }\end{array}$ \\
\hline 16224 & $\mathrm{~T} \rightarrow \mathrm{C}$ & 2 & $0 / 0 / \mathbf{1 0 7} / 1760$ & Oral cancer & 1.00 & $\begin{array}{l}\text { Rare, Japan, American, Finland, } \\
\text { Ashkenazi Jews }\end{array}$ \\
\hline 16230 & $\mathrm{~A} \rightarrow \mathrm{G}$ & 1 & $1854 / \mathbf{1 3} / 0 / 0$ & Polymorphism & 0.279 & $\begin{array}{l}\text { Extremely rare, South Asia, } \\
\text { Africa }\end{array}$ \\
\hline 16235 & $\mathrm{~A} \rightarrow \mathrm{G}$ & 2 & $1846 / 21 / 0 / 0$ & Oral cancer & 0.097 & Extremely rare, Africa, Italy \\
\hline 16249 & $\mathrm{~T} \rightarrow \mathrm{C}$ & 1 & 0/0/87/1780 & Prostate tumor & 0.720 & Rare, Africa, Japan, Italy \\
\hline 16256 & $\mathrm{C} \rightarrow \mathrm{T}$ & 2 & $0 / 0 / 1838 / 29$ & Oral cancer & 0.159 & Very rare, Asia, Finland, India \\
\hline 16261 & $\mathrm{C} \rightarrow \mathrm{T}$ & 2 & 0/0/1756/111 & Oral cancer & 1.00 & Japan, Taiwan, Finland, India \\
\hline 16269 & $\mathrm{~A} \rightarrow \mathrm{G}$ & 1 & $1855 / \mathbf{1 2} / 0 / 0$ & Polymorphism & 0.262 & Extremely rare, Japan, Italy \\
\hline 16270 & $\mathrm{C} \rightarrow \mathrm{T}$ & 3 & $0 / 0 / 1802 / 65$ & Oral cancer & 0.204 & Very rare, Finland, Italy, India \\
\hline 16278 & $\mathrm{C} \rightarrow \mathrm{T}$ & 1 & $0 / 0 / 1724 / \mathbf{1 4 3}$ & Oral cancer & 0.251 & Rare, Africa, Japan, Italy, India \\
\hline 16291 & $\mathrm{C} \rightarrow \mathrm{T}$ & 1 & $0 / 3 / 1816 / 48$ & Polymorphism & 1.00 & Very rare, Asia, Italy, Australia \\
\hline 16292 & $\mathrm{C} \rightarrow \mathrm{T}$ & 2 & $0 / 2 / 1801 / 64$ & $\begin{array}{l}\text { Breast, ovarian, head/neck tumor, } \\
\text { oral tumor }\end{array}$ & 0.663 & Very rare, Japan, Finland, Italy \\
\hline 16293 & $\mathrm{~A} \rightarrow \mathrm{G}$ & 1 & $1848 / \mathbf{1 7} / 2 / 0$ & Glioblastoma & 0.344 & $\begin{array}{l}\text { Extremely rare, Africa, Italy, } \\
\text { India }\end{array}$ \\
\hline 16294 & $\mathrm{C} \rightarrow \mathrm{T}$ & 2 & 0/0/1760/107 & Oral cancer & 1.00 & $\begin{array}{l}\text { Rare, Japan, India, Finland, } \\
\text { American }\end{array}$ \\
\hline 16296 & $\mathrm{C} \rightarrow \mathrm{T}$ & 1 & $0 / 0 / 1823 / 44$ & Polymorphism & 1.00 & $\begin{array}{l}\text { Very rare, Japan, American, } \\
\text { Finland }\end{array}$ \\
\hline 16304 & $\mathrm{~T} \rightarrow \mathrm{C}$ & 1 & 0/0/140/1727 & $\begin{array}{l}\text { Esophageal, breast and prostate } \\
\text { tumors, oral cancer }\end{array}$ & 0.252 & Asia, America, Italy \\
\hline 16311 & $\mathrm{~T} \rightarrow \mathrm{C}$ & 6 & $0 / 0 / 340 / 1526$ & Oral cancer & 0.554 & $\begin{array}{l}\text { Africa, Japan, China, } \\
\text { American, Finland, Italy, India }\end{array}$ \\
\hline
\end{tabular}


Table III. Continued.

\begin{tabular}{|c|c|c|c|c|c|c|}
\hline $\begin{array}{l}\text { mtDNA } \\
\text { position } \\
(\mathrm{CRS})\end{array}$ & Polymorphism & No & $\begin{array}{l}\mathrm{A} / \mathrm{G} / \mathrm{C} / \mathrm{T} / \mathrm{del} \\
\text { frequency } \\
(\mathrm{mtDB})\end{array}$ & $\begin{array}{l}\text { Tissues where } \\
\text { sequence was found } \\
\text { (MITOMAP) }\end{array}$ & P-value & $\begin{array}{l}\text { Region/population where } \\
\text { sequence variant } \\
\text { predominantly was found }\end{array}$ \\
\hline 16325 & $\mathrm{~T} \rightarrow \mathrm{C}$ & 2 & $0 / 0 / 47 / 1820$ & Polymorphism & 0.312 & $\begin{array}{l}\text { Very rare, Asia, Native } \\
\text { American, South America }\end{array}$ \\
\hline 16327 & $\mathrm{C} \rightarrow \mathrm{T}$ & 1 & $0 / 0 / 1843 / 24$ & Oral cancer & 0.443 & Extremely rare, Africa, Asia \\
\hline 16343 & $A \rightarrow G$ & 2 & $1852 / \mathbf{1 4} / 1 / 0$ & Polymorphism & 0.051 & $\begin{array}{l}\text { Extremely rare, Africa, } \\
\text { Melanesia, Polynesia }\end{array}$ \\
\hline 16354 & $\mathrm{C} \rightarrow \mathrm{T}$ & 1 & $0 / 0 / 1862 / 5$ & Polymorphism & 0.131 & $\begin{array}{l}\text { Extremely rare, Melanesia, } \\
\text { China, Finland }\end{array}$ \\
\hline 16362 & $\mathrm{~T} \rightarrow \mathrm{C}$ & 1 & $1 / 0 / 444 / 1422$ & Oral cancer & $0.001 \mathrm{U}$ & Africa, China, Japan, Italy \\
\hline 16390 & $\mathrm{G} \rightarrow \mathrm{A}$ & 1 & 62/1805/0/0 & Breast, ovarian tumor, oral cancer & 1.00 & $\begin{array}{l}\text { Very rare, Africa, Asia, } \\
\text { Ashkenazi Jew }\end{array}$ \\
\hline 16391 & $\mathrm{G} \rightarrow \mathrm{A}$ & 1 & $17 / 1850 / 0 / 0$ & Oral cancer, ovarian tumor & 0.344 & Extremely rare, Finland \\
\hline 16482 & $\mathrm{~A} \rightarrow \mathrm{G}$ & 1 & $1864 / 3 / 0 / 0$ & Polymorphism & 0.089 & Extremely rare, Italy \\
\hline 16519 & $\mathrm{~T} \rightarrow \mathrm{C}$ & 16 & 0/0/1115/752 & $\begin{array}{l}\text { Oral cancer, gastric, lung, ovarian } \\
\text { tumor }\end{array}$ & $0.003 \mathrm{U}$ & $\begin{array}{l}\text { Africa, Japan, Caucasian, } \\
\text { China, American, Finland, } \\
\text { Italy, India }\end{array}$ \\
\hline 16526 & $\mathrm{G} \rightarrow \mathrm{A}$ & 2 & 19/1848/0/0 & Polymorphism & 0.083 & $\begin{array}{l}\text { Extremely rare, Asia, } \\
\text { Finland }\end{array}$ \\
\hline
\end{tabular}

U, polymorphism under-represented in breast cancer population; O, polymorphism over-represented in breast cancer population.

OR=2.4583, which means that a $7028 \mathrm{C}$ carrier had a 2.45 higher chance not to develop cancer with specificity 0.636 (0.513-0.747). The cancer-free status positive predictive value (precision rate PPV) of $7025 \mathrm{C}$ test is 0.787 (0.714-0.851 at $95 \% \mathrm{CI}$ ), posterior probability (odds): $79 \%$, with relative risk reduction $\mathrm{RRR}=-0.311$ and number needed to diagnose $\mathrm{NND}=4.53$.

Inherited haplogroup-related polymorphisms analyzed shown no correlation with TNM or clinical stage. At this time it is probably the effect of lack of effective populationvide screening annual programs. TNM/stage at detection is more dependent on public awareness of early detection's, access to detection and diagnostic centers and organization of screening programs in place which seem not to be satisfactory (63).

D-loop polymorphisms: C-tract insertions in breast cancer patients. There were two common insertion polymorphisms seen in breast cancer patients. The first is the insertion of a single cytosine $(C)$ in the poly-C tract spanning nucleotide positions 310-315. The reference sequence contains a run of 5 consecutive $\mathrm{Cs}$, while the expansion makes this a run of $6 \mathrm{Cs}$. We found this $5 \mathrm{C}-6 \mathrm{C}$ insertion event in 32 breast cancer patients. The second similar polymorphism is also a single $\mathrm{C}$ insertion, but in the $7 \mathrm{C}$ run between nucleotide positions $303-310$. We found this $7 \mathrm{C}-8 \mathrm{C}$ variant in 15 patients. Of these 15 patients, 14 also had the single $\mathrm{C}$ insertion in the 310-315 tract, while a single patient had the $\mathrm{C}$ insertion at the 303-310 position without the corresponding insertion in the 310-315 position. In other words, the insertion of one $\mathrm{C}$ in the 303-315 tract is almost always accompanied by a second insertion in the 303-310 tract. Of the 32 patients with the 310-315 insertion, 14 also had the insertion in the 303-310 tract. Thus, an insertion is the $310-315$ tract is accompanied by a second insertion in the $303-310$ tract $44 \%$ of the time.

D-loop SNPs specific found for breast cancer. As outlined in the methods section, 1120 bases of the D-loop were sequenced in all breast cancer patients (spanning nucleotide positions 16024 to 576) and differences from the reference Cambridge sequence were identified and their frequency compared to the frequency in the online searchable database mtDB. A complete list of all changes and their statistical analysis is listed in Table III. There were only a small number of SNPs that reached statistical significance. These included 7 SNPs that were seen at significantly lower frequency in breast cancer cases compared to the diverse population represented in mtDB (A73G, C150T, T16183C, T16189C, C16223T, T16362C, T16519C) and 3 that were overrepresented in the breast cancer patient group (T239C, A263G, and C16207T).

\section{Discussion}

The fact that family history is a risk factor for breast cancer suggests possible inherited genetic susceptibility. There is broad agreement that there are many genes involved in the process of breast carcinogenesis and that risk for breast cancer increases with each relevant genetic alteration present. In particular, breast cancer development is associated with 


\section{D-loop mtDNA polymorphisms distribution in breast cancer patients (1)}

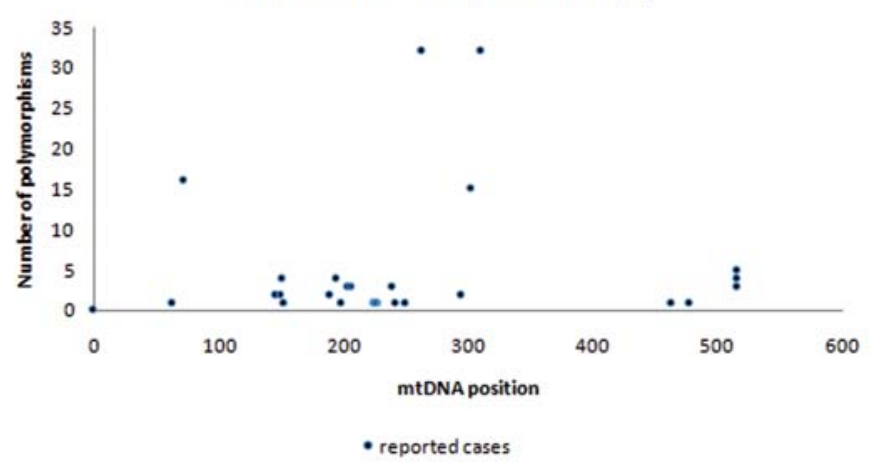

\section{D-loop mtDNA polymorphisms distribution in breast cancer patients (2)}

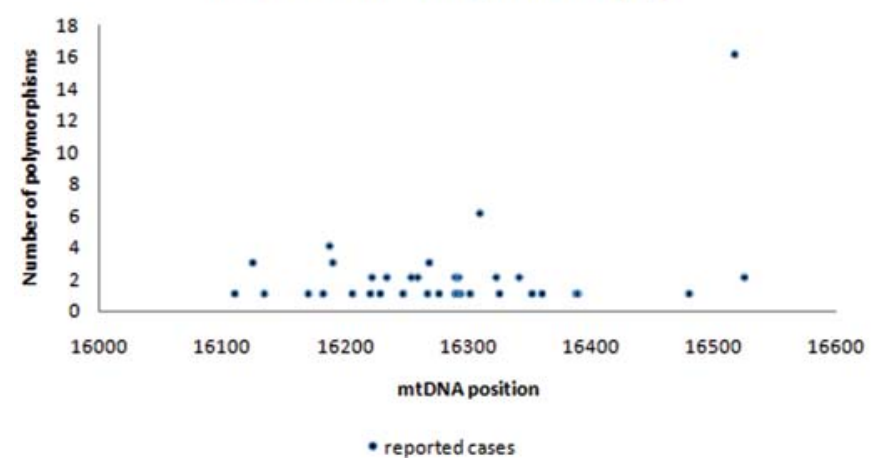

Figure 3. mtDNA D-loop polymorphisms distribution pattern in breast cancer cohort.

autosomal dominant inheritance of mutated BRCA1 or BRCA2 genes. Despite the established role of several genes important in breast cancer, the list of genetic factors involved in this disease is still incomplete and more effort is needed to identify biomarkers and genetic alterations that would allow the selection of individuals with increased risk for intensive screening, prevention and treatment programs $(1,10,17)$.

The use of mitochondrial DNA polymorphisms as a biomarker is rapidly expanding in metabolic diseases, aging, cancer, the analysis of human migration patterns, and human identification in forensic sciences (20-22). In breast cancer mtDNA analysis seems to be an attractive clinical target, as mtDNA mutations and/or polymorphisms can be detected in the material obtained from serum and WBC (64) and also in nipple aspirate fluid $(23,65)$ and ductal lavage $(66)$. So far, the prevalence of mtDNA mutations in human BCs has been analyzed mostly in patient cohorts recruited from China and Taiwan $(24,67,68)$, India $(69,70)$, or African-Americans $(71,72)$ but no large-scale research included Europeans or Caucasians $(21,33,37)$.

Tumor-associated mitochondrial genomic instability is seen in breast cancer patients and the presence of one or more somatic point mutations is found in $29 \%$ (73) to $93 \%$ of patients (74). The mononucleotide repeat (D310) appeared as a mutation 'hot-spot' in primary tumors $(21,31,33)$.
In sporadic $\mathrm{BC}$ the inherited $10398 \mathrm{G}$ polymorphism was associated with susceptibility to BC $(35,75)$. In familial breast cancer inheritance of G9055A, A10398G, T16519C polymorphisms were shown to increase breast cancer risk, while T3197C and G13708A decreased breast cancer development risk (76). Other patient populations were shown to carry different patterns of mtDNA mutations including changes not only in the D-loop, but also ND5, ND1, ND4, CYTB (77) or ATPase8, ATPase6, COII, ND5 (24) genes. To date, no correlation between the pattern of mitochondrial abnormalities and clinical or histopathological features has been found (73).

Our analysis of the D-loop revealed no known pathogenic mtDNA mutations but multiple polymorphisms, including microsatellite instability in the ' $\mathrm{C}$-tract' (between nucleotides 303 and 315$)$ in $32(73 \%)$ of BC patients. We did not detect any somatic D-loop mtDNA mutations which is different from what some others have reported $(23,73)$. We believe that this is a result of the stringent quality control we used $(34,78)$, similar to the mitochondrial breast cancer project by Wang et al, who also reported no somatic mutations in the patient cohort (79). As previously pointed out by Salas et al (80) mtDNA sequencing and analysis techniques contain inherent problems, particularly with regard to the generation of authentic and useful data. It is important to employ standardized procedures based on scientific grounds, in order to have mtDNA-based evidence that can be accepted in clinical practice. Therefore, we have put much effort into the refinement of our amplification and sequencing strategies, as well as quality control of mtDNA sequencing $(34,35,81)$. We believe that careful analysis of possible technical errors at any stage of the analysis, DNA extraction, amplification, mutation screening, mtDNA sequencing, and patient documentation may prevent misinterpretation and we have put much effort in quality control (82). To further increase specificity of our mutation screen, we took advantage of detailed multiple-database analysis, as it has proven to be an effective and successful method of data evaluation for mtDNA $(78,80,83)$. Our summary polymorphism table should be treated and interpreted judiciously, as previously reported cancer mutations may have arisen from technical errors in previous projects $(83,84)$.

Multiple interesting mtDNA polymorphisms and mutations exist in prostate (35) thyroid (92) and oral cancers (39). Thus, others have hypothesized that patients bearing some of the common mitochondrial polymorphisms are at higher risk of cancer development. Also some of the mtDNA variants reported as possible tumor-specific somatic mtDNA mutations are actually common in the general population and proposed therefore a paradigm has been proposed for evaluation of sequence quality and for detection of potential problems with inferring a pathogenic status of a particular mutation and to avoid false positive results $(20,22,87)$. At the same time, some mtDNA mutations and polymorphisms may develop before cell transformation and may be found in precancerous tissues (88) including tumor margins, as it is in the case of TP53 mutations in lung cancer (89). The possibility of pre-cancerous mtDNA variation should also be considered in experiment planning and data interpretation to avoid false negative results (34). 
Because the complement of germline polymorphisms in cancer patients and controls are different, it is possible that the inheritance of specific mitochondrial genotypes predisposes individuals to cancer $(90,91)$. Moreover, the co-occurrence of multiple inherited mtDNA SNPs may influence the disease phenotype as in the case of $12308 \mathrm{G}$ and $10398 \mathrm{G}$ in BC (62) or G10680A and T14484C in Leber hereditary optic neuropathy (LHON) (92). One polymorphism may have a potential modifier role in increasing the penetrance and expressivity of other mtDNA genome alternations. In particular, the mitochondrial haplotypes may play a synergistic role in the development of cancer as is the case of vision loss in the families carrying the LHON-associated primary mtDNA mutations. In LHON patients the mitochondrial haplotype has been shown to influence the clinical expression (93). Therefore, our project covers haplogroup related polymorphisms as BC biomarkers. Our strictly European population is exclusively distributed among the nine haplogroups designated: H, I, J, K, T, U, V, W, and X, and no analysis of haplogroups characteristic for Asian or African populations was included (54). Our research was also informed by the fact that a significant increased risk in breast cancer development was reported for haplogroup K (76). We have also chosen to focus on haplogroup-specific polymorphisms as we and others have previously correlated A10398G polymorphism characteristic for haplogroup I, J, K with BC development $(35,70,71,75,94)$. The BC cohort haplogroup distribution was different from the healthy Polish population and cancer-free centenarians with haplogroup $\mathrm{H}$ underrepresented in $\mathrm{BC}$ patients. We believe that this might suggest a protective role of haplogroup $\mathrm{H}$ in cancer development. Our hypothesis seems plausible as mtDNA haplogroup $\mathrm{H}$ is very common in Caucasoids, reaching frequencies of $\sim 50 \%$. Recent studies have suggested haplogroup H3 is highly protective against AIDS progression (95). Moreover, mtDNA haplogroup $\mathrm{H}$ is a strong independent predictor of outcome during severe sepsis (96).

Our polymorphism analysis also focused on the D-loop region of mtDNA, as it contains two hypervariable regions: HV1 (16024-16383) and HV2 (57-333) which were reported as somatic mutation 'hot spots' in many types of cancer including sequence length poly-C polymorphisms $(21,97)$. Breast cancer patients with multiple mtDNA D-loop (CA) (n) polymorphisms have significantly poorer disease-free survival than those with one copy of the mtDNA D-loop (CA) (n) polymorphism (98). We also believe that mtDNA nucleotide variations, particularly those in the D310 segment, might be involved in the breast carcinogenesis and could be included in a panel of molecular biomarkers for cancer susceptibility or early-detection strategies. In particular, C16189T could be of interest as this mtDNA variant has been associated with susceptibility to dilated cardiomyopathy (DCM) (99), insulin resistance and development of diabetes mellitus (DM) in adult life and vascular pathologies involved in stroke (lacunar cerebral infarction) and cardiovascular diseases (100).

Precise understanding of the molecular mechanisms whereby specific mtDNA polymorphisms predispose to cancer is currently lacking. Although the mechanisms of generation and functional impact of mtDNA polymorphisms are still not clear, there is a high incidence and broad distribution in human cancers making them a potential marker for cancer detection, but the role of mtDNA polymorphisms in the maintenance of tumor cell phenotype or in tumorigenesis remains to be elucidated $(21,22,31,33,101,102)$. Although it is generally believed that polymorphisms may have pathogenic potential, it remains to be established whether these polymorphisms contribute to neoplastic transformation by changing cellular energy capacities, increasing mitochondrial oxidative stress, or modulating apoptosis. The significance of each individual polymorphism for mitochondrial function and tumorigenesis is unknown and only functional analysis in relation to cell behavior, proliferation, and apoptosis can determine the significance of these polymorphisms and their association with tumorigenesis. We believe that polymorphisms in mtDNA, both in the D-loop and in the coding region (including haplogroup specific positions) may cause subtle differences in the encoded protein structure and function (40). Particular mtDNA genotypes may predispose an individual to an earlier onset of degenerative cellular processes, such as the accumulation of somatic mtDNA variation, decline in OXPHOS capacity or faster cancer progression, as shown in transmitochondrial cybrids (103). The mtDNA haplotype may also influence its further mutagenesis, as has been shown for haplogroup $\mathrm{J}$ that predisposes the mitochondrial genome to mutate at locus 14484 , possibly through nearneighbor effects as previously described to occur during mtDNA evolution (104).

Finally, from a broader perspective we believe that cancer control efforts in the postgenome era should be focused at both population and individual levels to develop novel risk assessment and treatment strategies that will further reduce the morbidity and mortality associated with the disease. The discovery that mutations in particular genes increase the risk of breast cancer has radically transformed our understanding of the pathology of breast cancer. Moreover, a better understanding of tumor biology may lead to improvements in the multidisciplinary management of breast cancer, and its pathologic evaluation if routine clinical examination was enhanced with molecular analysis. A number of genetic biomarkers should be developed for routine use. The immediate challenge now is to learn how to use the molecular characteristics of an individual to improve early detection and ultimately to decrease breast cancer morbidity $(1,18,34,37)$.

The inherited mitochondrial genotype of women with breast cancer differs from that of multiple comparison groups including a rigorously defined no-cancer control group, the general Polish population, and a group of Polish centenarians. This is documented by differences in the standard mitochondrial haplotype analysis (haplogroups) as well as SNPs in the highly variable D-loop. This finding suggests that inherited mtDNA variation may predispose to or protect from the development of breast cancer in women. We found no somatically acquired or tumor-specific mutations in the D-loop area of the mitochondrial genome, a finding that is consistent with at least one other report, but is at odds with other reports, perhaps due to our more stringent and redundant analysis of DNA sequence. The identification of specific SNPs that are over- and underrepresented in cancer patients suggests the possibility of their application 
in identifying high risk groups for the clinical care of women, particularly in the areas of screening and detection, though the utility of these genetic markers has not been tested in this circumstance. Our results are similar to findings in other tumor types, especially prostate and renal cancers, where inherited mitochondrial genotype has been shown to vary significantly between individuals with cancer and no-cancer controls.

\section{Acknowledgements}

This study was supported by the Ministry of Science and Higher Education of The Republic of Poland Grant No. N N401 232733 to E.B. and A.M.C.; Oligo.pl Minigrant G11 to E.B., P.G., A.M.C. and K.P., Polish Genetics Society Grant 2006/07 to E.B., A.M.C., K.P. and A.K. A.M.C. was supported by FEBS Collaborative Experimental Scholarship for Central \& Eastern Europe, Fulbright Junior Research Grant and The Kosciuszko Foundation Scholarship. The project realisation by its authors would not have been possible without the support of Professor Piotr Weglenski, PhD (Institute of Genetics and Biotechnology, Faculty of Biology, University of Warsaw). The authors would like to thank Jerzy S. Czarnecki, PhD (University of Lodz, Lodz, Poland) and Przemyslaw Tomalski, PhD (Centre for Brain and Cognitive Development, School of Psychology, Birkbeck College, UK) for critical reading of the manuscript and fruitful discussions.

\section{References}

1. Olopade OI, Grushko TA, Nanda R and Huo D: Advances in breast cancer: pathways to personalized medicine. Clin Cancer Res 14: 7988-7999, 2008.

2. Jemal A, Siegel R, Ward E, et al: Cancer statistics, 2006. CA Cancer J Clin 56: 106-130, 2006.

3. Jemal A, Siegel R, Ward E, Murray T, Xu J and Thun MJ: Cancer statistics, 2007. CA Cancer J Clin 57: 43-66, 2007.

4. Levi F, Lucchini F, Negri E and La Vecchia C: Continuing declines in cancer mortality in the European Union. Ann Oncol 18: 593-595, 2007.

5. Balleine RL, Webster LR, Davis S, et al: Molecular grading of ductal carcinoma in situ of the breast. Clin Cancer Res 14: 8244-8252, 2008.

6. Geyer FC, Marchio C and Reis-Filho JS: The role of molecular analysis in breast cancer. Pathology 41: 77-88, 2009.

7. Welcsh PL and King MC: BRCA1 and BRCA2 and the genetics of breast and ovarian cancer. Hum Mol Genet 10: 705-713, 2001

8. Foulkes WD and Narod SA: Hereditary breast and ovarian cancer: epidemiology, genetics, screening and predictive testing. Clin Invest Med 18: 473-483, 1995

9. Wideroff L, Vadaparampil ST, Greene MH, Taplin S, Olson L and Freedman AN: Hereditary breast/ovarian and colorectal cancer genetics knowledge in a national sample of US physicians. J Med Genet 42: 749-755, 2005.

10. Lubinski J, Korzen M, Gorski B, et al: Breast cancer susceptibility genes. J BUON 12 (Suppl 1.): S23-S29, 2007.

11. Jakubowska A, Jaworska K, Cybulski C, et al: Do BRCA1 modifiers also affect the risk of breast cancer in non-carriers? Eur J Cancer 45: 837-842, 2009.

12. Antoniou AC, Cunningham AP, Peto J, et al: The BOADICEA model of genetic susceptibility to breast and ovarian cancers: updates and extensions. Br J Cancer 98: 1457-1466, 2008.

13. Walsh T, Casadei S, Coats KH, et al: Spectrum of mutations in BRCA1, BRCA2, CHEK2, and TP53 in families at high risk of breast cancer. JAMA 295: 1379-1388, 2006.

14. Debniak T, Scott RJ, Huzarski T, et al: XPD common variants and their association with melanoma and breast cancer risk. Breast Cancer Res Treat 98: 209-215, 2006.
15. Simpson S: PALB2-new breast-cancer susceptibility gene. Lancet Oncol 8: 105, 2007.

16. Garcia-Closas M and Chanock S: Genetic susceptibility loci for breast cancer by estrogen receptor status. Clin Cancer Res 14: 8000-8009, 2008

17. Lubinski J, Korzen M, Gorski B, et al: Genetic contribution to all cancers: the first demonstration using the model of breast cancers from Poland stratified by age at diagnosis and tumour pathology. Breast Cancer Res Treat 114: 121-126, 2009.

18. Pietka G, Kukwa W, Bartnik E, Scinska A and Czarnecka AM: [Mitochondrial DNA mutations in the pathogenesis in the head and neck squamous cell carcinoma]. Otolaryngol Pol 62: 158-164, 2008.

19. Plak K, Kukwa W, Bartnik E, et al: [The impact of mtDNA mutations on proteins structure in selected types of cancer]. Postepy Biochem 54: 151-160, 2008.

20. Brandon M, Baldi P and Wallace DC: Mitochondrial mutations in cancer. Oncogene 25: 4647-4662, 2006.

21. Czarnecka A, Golik P and Bartnik E: Mitochondrial DNA mutations in human neoplasia. J Appl Genet 47: 67-78, 2006.

22. Wallace DC: A mitochondrial paradigm of metabolic and degenerative diseases, aging, and cancer: a dawn for evolutionary medicine. Annu Rev Genet 39: 359-407, 2005.

23. Zhu W, Qin W, Bradley P, Wessel A, Puckett CL and Sauter ER: Mitochondrial DNA mutations in breast cancer tissue and in matched nipple aspirate fluid. Carcinogenesis 26: 145-152, 2005.

24. Tseng LM, Yin PH, Chi CW, et al: Mitochondrial DNA mutations and mitochondrial DNA depletion in breast cancer. Genes Chromosomes Cancer 45: 629-638, 2006.

25. Elmore JG, Barton MB, Moceri VM, Polk S, Arena PJ and Fletcher SW: Ten-year risk of false positive screening mammograms and clinical breast examinations. N Engl J Med 338: 1089-1096, 1998

26. NIH: National Institutes of Health Consensus Development Conference Statement: breast cancer screening for women ages 40-49, January 21-23, 1997. National Institutes of Health Consensus Developmental Panel. J Natl Cancer Inst Monogr: vii-xviii, 1997.

27. Berg WA, Blume JD, Cormack JB, et al: Combined screening with ultrasound and mammography vs mammography alone in women at elevated risk of breast cancer. JAMA 299: 2151-2163, 2008.

28. Tyrer J, Duffy SW and Cuzick J: A breast cancer prediction model incorporating familial and personal risk factors. Stat Med 23: 1111-1130, 2004

29. Lawson HW, Henson R, Bobo JK and Kaeser MK: Implementing recommendations for the early detection of breast and cervical cancer among low-income women. Oncology (Williston Park) 14: 1528-1530, 1638, 1641-1522 passim, 2000.

30. Czarnecka AM, Marino Gammazza A, Di Felice V, Zummo G and Cappello F: Cancer as a 'Mitochondriopathy'. J Cancer Mol 3: 71-79, 2007

31. Petros JA, Baumann AK, Ruiz-Pesini E, et al: mtDNA mutations increase tumorigenicity in prostate cancer. Proc Natl Acad Sci USA 102: 719-724, 2005.

32. Ishikawa $\mathrm{K}$, Takenaga $\mathrm{K}$, Akimoto $\mathrm{M}$, et al: ROS-generating mitochondrial DNA mutations can regulate tumor cell metastasis. Science 320: 661-664, 2008

33. Czarnecka AM and Bartnik E: Mitochondrial DNA mutations in tumors. In: Cellular Respiration and Carcinogenesis. Apte SP and Sarangarajan R (eds). Humana Press, New York, pp1-12, 2009.

34. Czarnecka AM, Krawczyk T, Czarnecki JS, et al: Methodology for mitochondrial DNA research in oncology: goals and pitfalls. ARS Medica Tomitana XIV: pp48-64, 2008.

35. Czarnecka AM, Krawczyk T, Zdrozny M, et al: Mitochondrial NADH-dehydrogenase subunit 3 (ND3) polymorphism (A10398G) and sporadic breast cancer in Poland. Breast Cancer Res Treat: 511-518, 2010.

36. Klemba A, Kukwa W, Bartnik E, et al: [Molecular biology of endometrial carcinoma]. Postepy Hig Med Dosw (Online) 62: 420-432, 2008.

37. Plak K, Czarnecka AM, Krawczyk T, Golik P and Bartnik E: Breast cancer as a mitochondrial disorder (Review). Oncol Rep 21: 845-851, 2009.

38. Aikhionbare FO, Khan M, Carey D, Okoli J and Go R: Is cumulative frequency of mitochondrial DNA variants a biomarker for colorectal tumor progression? Mol Cancer 3: 30, 2004. 
39. Datta S, Majumder M, Biswas NK, Sikdar N and Roy B: Increased risk of oral cancer in relation to common Indian mitochondrial polymorphisms and autosomal GSTP1 locus. Cancer 110: 1991-1999, 2007.

40. Singh KK and Kulawiec M: Mitochondrial DNA polymorphism and risk of cancer. Methods Mol Biol 471: 291-303, 2009.

41. Ha PK, Tong BC, Westra WH, et al: Mitochondrial C-tract alteration in premalignant lesions of the head and neck: a marker for progression and clonal proliferation. Clin Cancer Res 8: 2260-2265, 2002.

42. Fliss MS, Usadel H, Caballero OL, et al: Facile detection of mitochondrial DNA mutations in tumors and bodily fluids. Science 287: 2017-2019, 2000 .

43. Piechota J, Tonska K, Nowak M, Kabzinska D, Lorenc A and Bartnik E: Comparison between the Polish population and European populations on the basis of mitochondrial morphs and haplogroups. Acta Biochim Pol 51: 883-895, 2004.

44. Ingman M and Gyllensten U: mtDB: Human Mitochondrial Genome Database, a resource for population genetics and medical sciences. Nucleic Acids Res 34: D749-D751, 2006.

45. Ruiz-Pesini E, Lott MT, Procaccio V, et al: An enhanced MITOMAP with a global mtDNA mutational phylogeny. Nucleic Acids Res 35: D823-D828, 2007.

46. Tavassoli FA and Devilee P: World Health Organisation Classification of Tumours: Pathology and genetics of tumours of the breast and female genital organs. IARC Press-WHO, 2003

47. Mossakowska M, Barcikowska M, Broczek K, et al: Polish Centenarians Programme. Multidisciplinary studies of successful ageing: aims, methods, and preliminary results. Exp Gerontol 43: 238-244, 2008

48. Jakubowska A, Gronwald J, Menkiszak J, et al: Ovarian cancer risk in Polish BRCA1 mutation carriers is not associated with the prohibitin 3' untranslated region polymorphism. BMC Cancer 8: 90, 2008.

49. Booker LM, Habermacher GM, Jessie BC, et al: North American white mitochondrial haplogroups in prostate and renal cancer. J Urol 175: 468-472, 2006.

50. Andrews RM, Kubacka I, Chinnery PF, Lightowlers RN, Turnbull DM and Howell N: Reanalysis and revision of the Cambridge reference sequence for human mitochondrial DNA. Nat Genet 23: 147, 1999.

51. Wiesbauer M, Meierhofer D, Mayr JA, Sperl W, Paulweber B and Kofler B: Multiplex primer extension analysis for rapid detection of major European mitochondrial haplogroups. Electrophoresis 27: 3864-3868, 2006.

52. Finnila S, Lehtonen MS and Majamaa K: Phylogenetic network for European mtDNA. Am J Hum Genet 68: 1475-1484, 2001 .

53. Macaulay V, Richards M, Hickey E, et al: The emerging tree of West Eurasian mtDNAs: a synthesis of control-region sequences and RFLPs. Am J Hum Genet 64: 232-249, 1999.

54. Torroni A, Huoponen K, Francalacci P, et al: Classification of European mtDNAs from an analysis of three European populations. Genetics 144: 1835-1850, 1996.

55. Thompson JD, Higgins DG and Gibson TJ: CLUSTAL W: improving the sensitivity of progressive multiple sequence alignment through sequence weighting, position-specific gap penalties and weight matrix choice. Nucleic Acids Res 22: 4673-4680, 1994.

56. Anderson S, Bankier AT, Barrell BG, et al: Sequence and organization of the human mitochondrial genome. Nature 290: 457-465, 1981

57. Sheskin DJ: Handbook of parametric and non-parametric statistical procedures. Chapman \& Hall, Boca Raton, FL, 2007.

58. Campbell I: Chi-squared and Fisher-Irwin tests of two-bytwo tables with small sample recommendations. Stat Med 26: 3661-3675, 2007.

59. Fleiss JL, Levin B, Cho Paik M and Fleiss J: Statistical methods for rates and proportions. Wiley-Interscience Inc., San Francisco, CA, 2003.

60. Rosner B: Fundamentals of Biostatistics. Duxbury Press, Florence, KY 2005.

61. Malyarchuk BA, Grzybowski T, Derenko MV, Czarny J Wozniak M and Miscicka-Sliwka D: Mitochondrial DNA variability in Poles and Russians. Ann Hum Genet 66: 261-283, 2002

62. Covarrubias D, Bai RK, Wong LJ and Leal SM: Mitochondrial DNA variant interactions modify breast cancer risk. J Hum Genet 53: 924-928, 2008.
63. Borella L: [The European countries confronting cancer: a set of indicators assessing public health status]. Bull Cancer 95: 1053-1062, 2008.

64. Losanoff JE, Zhu W, Qin W, Mannello F and Sauter ER: Can mitochondrial DNA mutations in circulating white blood cells and serum be used to detect breast cancer? Breast 17: 540-542, 2008.

65. Jakupciak JP, Maggrah A, Maragh S, et al: Facile whole mitochondrial genome resequencing from nipple aspirate fluid using MitoChip v2.0. BMC Cancer 8: 95, 2008

66. Isaacs C, Cavalli LR, Cohen Y, et al: Detection of LOH and mitochondrial DNA alterations in ductal lavage and nipple aspirate fluids from high-risk patients. Breast Cancer Res Treat 84: 99-105, 2004.

67. Tan DJ, Bai RK and Wong LJ: Comprehensive scanning of somatic mitochondrial DNA mutations in breast cancer. Cancer Res 62: 972-976, 2002

68. Tseng LM, Yin PH, Tsai YF, et al: Association between mitochondrial DNA 4,977 bp deletion and NAD(P)H:quinone oxidoreductase 1 C609T polymorphism in human breast tissues. Oncol Rep 21: 1169-1174, 2009.

69. Gochhait S, Bhatt A, Sharma S, Singh YP, Gupta P and Bamezai RN: Concomitant presence of mutations in mitochondrial genome and p53 in cancer development, a study in north Indian sporadic breast and esophageal cancer patients. Int J Cancer 123: 2580-2586, 2008.

70. Bhat A, Koul A, Sharma S, et al: The possible role of $10398 \mathrm{~A}$ and $16189 \mathrm{C}$ mtDNA variants in providing susceptibility to T2DM in two North Indian populations: a replicative study. Hum Genet 120: 821-826, 2007.

71. Canter JA, Kallianpur AR, Parl FF and Millikan RC: Mitochondrial DNA G10398A polymorphism and invasive breast cancer in African-American women. Cancer Res 65: 8028-8033, 2005.

72. Mims MP, Hayes TG, Zheng S, et al: Mitochondrial DNA G10398A polymorphism and invasive breast cancer in AfricanAmerican women. Cancer Res 66: 1880; author reply 1880-1881, 2006.

73. Wang Y, Liu VW, Tsang PC, et al: Microsatellite instability in mitochondrial genome of common female cancers. Int J Gynecol Cancer 16 (Suppl. 1): 259-266, 2006.

74. Zhou S, Kachhap S, Sun W, et al: Frequency and phenotypic implications of mitochondrial DNA mutations in human squamous cell cancers of the head and neck. Proc Natl Acad Sci USA 104: 7540-7545, 2007.

75. Pezzotti A, Kraft P, Hankinson SE, Hunter DJ, Buring J and Cox DG: The mitochondrial A10398G polymorphism, interaction with alcohol consumption, and breast cancer risk. PLoS ONE 4: e5356, 2009.

76. Bai RK, Leal SM, Covarrubias D, Liu A and Wong LJ: Mitochondrial genetic background modifies breast cancer risk. Cancer Res 67: 4687-4694, 2007.

77. Parrella P, Xiao Y, Fliss M, et al: Detection of mitochondrial DNA mutations in primary breast cancer and fine-needle aspirates. Cancer Res 61: 7623-7626, 2001.

78. Salas A, Carracedo A, Macaulay V, Richards M and Bandelt HJ: A practical guide to mitochondrial DNA error prevention in clinical, forensic, and population genetics. Biochem Biophys Res Commun 335: 891-899, 2005.

79. Wang CY, Wang HW, Yao YG, Kong QP and Zhang YP: Somatic mutations of mitochondrial genome in early stage breast cancer. Int J Cancer 121: 1253-1256, 2007.

80. Salas A, Yao YG, Macaulay V, Vega A, Carracedo A and Bandelt HJ: A critical reassessment of the role of mitochondria in tumorigenesis. PLoS Med 2: e296, 2005.

81. Parson W and Bandelt HJ: Extended guidelines for mtDNA typing of population data in forensic science. Forensic Sci Int Genet 1: 13-19, 2007.

82. Bandelt HJ and Salas A: Contamination and sample mix-up can best explain some patterns of mtDNA instabilities in buccal cells and oral squamous cell carcinoma. BMC Cancer 9: 113, 2009.

83. Bandelt HJ, Yao YG, Salas A, Kivisild T and Bravi CM: High penetrance of sequencing errors and interpretative shortcomings in mtDNA sequence analysis of LHON patients. Biochem Biophys Res Commun 352: 283-291, 2007.

84. Bandelt HJ, Quintana-Murci L, Salas A and Macaulay V: The fingerprint of phantom mutations in mitochondrial DNA data. Am J Hum Genet 71: 1150-1160, 2002

85. Gomez-Zaera M, Abril J, Gonzalez L, et al: Identification of somatic and germline mitochondrial DNA sequence variants in prostate cancer patients. Mutat Res 595: 42-51, 2006. 
86. Maximo V, Soares P, Lima J, Cameselle-Teijeiro J and Sobrinho-Simoes M: Mitochondrial DNA somatic mutations (point mutations and large deletions) and mitochondrial DNA variants in human thyroid pathology: a study with emphasis on Hurthle cell tumors. Am J Pathol 160: 1857-1865, 2002.

87. Bandelt HJ, Yao YG, Bravi CM, Salas A and Kivisild T: Median network analysis of defectively sequenced entire mitochondrial genomes from early and contemporary disease studies. J Hum Genet 54: 174-181, 2009.

88. Tzen CY, Mau BL and Wu TY: ND4 mutation in transitional cell carcinoma: does mitochondrial mutation occur before tumorigenesis? Mitochondrion 7: 273-278, 2007.

89. Jassem J, Jassem E, Jakobkiewicz-Banecka J, et al: P53 and $\mathrm{K}$-ras mutations are frequent events in microscopically negative surgical margins from patients with non-small cell lung carcinoma. Cancer 100: 1951-1960, 2004.

90. Bandelt HJ, Salas A and Bravi CM: What is a 'novel' mtDNA mutation, and does 'novelty' really matter? J Hum Genet 51: 1073-1082, 2006

91. Raule N, Sevini F, Santoro A, Altilia S and Franceschi C: Association studies on human mitochondrial DNA: methodological aspects and results in the most common age-related diseases. Mitochondrion 7: 29-38, 2007.

92. Yang J, Zhu Y, Tong Y, et al: The novel G10680A mutation is associated with complete penetrance of the LHON/T14484C family. Mitochondrion 9: 273-278, 2009.

93. Ji Y, Zhang AM, Jia X, et al: Mitochondrial DNA haplogroups M7b1'2 and M8a affect clinical expression of leber hereditary optic neuropathy in Chinese families with the $\mathrm{m} .11778 \mathrm{G} \rightarrow \mathrm{a}$ mutation. Am J Hum Genet 83: 760-768, 2008.

94. Darvishi K, Sharma S, Bhat AK, Rai E and Bamezai RN Mitochondrial DNA G10398A polymorphism imparts maternal Haplogroup $\mathrm{N}$ a risk for breast and esophageal cancer. Cancer Lett 249: 249-255, 2007.
95. Hendrickson SL, Hutcheson HB, Ruiz-Pesini E, et al: Mitochondrial DNA haplogroups influence AIDS progression. AIDS 22: 2429-2439, 2008.

96. Baudouin SV, Saunders D, Tiangyou W, et al: Mitochondrial DNA and survival after sepsis: a prospective study. Lancet 366: 2118-2121, 2005.

97.Hibi K, Nakayama H, Yamazaki T, et al: Detection of mitochondrial DNA alterations in primary tumors and corresponding serum of colorectal cancer patients. Int J Cancer 94: 429-431, 2001

98. Ye C, Gao YT, Wen W, et al: Association of mitochondrial DNA displacement loop (CA)n dinucleotide repeat polymorphism with breast cancer risk and survival among Chinese women. Cancer Epidemiol Biomarkers Prev 17: 2117-2122, 2008.

99. Ruppert V, Nolte D, Aschenbrenner T, Pankuweit S, Funck R and Maisch B: Novel point mutations in the mitochondrial DNA detected in patients with dilated cardiomyopathy by screening the whole mitochondrial genome. Biochem Biophys Res Commun 318: 535-543, 2004.

100.Liou CW, Lin TK, Huang FM, et al: Association of the mitochondrial DNA $16189 \mathrm{~T}$ to $\mathrm{C}$ variant with lacunar cerebral infarction: evidence from a hospital-based case-control study. Ann N Y Acad Sci 1011: 317-324, 2004

101. Wallace DC: Mitochondrial diseases in man and mouse. Science 283: 1482-1488, 1999

102. Wallace DC: Mitochondria and cancer: Warburg addressed. Cold Spring Harb Symp Quant Biol 70: 363-374, 2005.

103. Munakata K, Tanaka M, Mori K, et al: Mitochondrial DNA $3644 \mathrm{~T} \rightarrow \mathrm{C}$ mutation associated with bipolar disorder. Genomics 84: $1041-1050,2004$

104. Elliott HR, Samuels DC, Eden JA, Relton CL and Chinnery PF: Pathogenic mitochondrial DNA mutations are common in the general population. Am J Hum Genet 83: 254-260, 2008. 